\title{
Caracterización clínica, histológica y endoscópica del cáncer gástrico en el Hospital de Especialidades Dr. Abel Gilbert Pontón, Ecuador
}

\section{Clinical, histological, and endoscopic characterization of gastric cancer at the Hospital de Especialidades Dr. Abel Gilbert Pontón, Ecuador}

\author{
Rubén Gustavo Muñoz-Cedeño, ${ }^{1 *}$ (1) Priscila Martínez, ${ }^{2}$ [] Viviana Paullán-Sani, ${ }^{3}$ (i) Gema Rodríguez-Chica. ${ }^{4}$ (])
}

\author{
Gacceso abierto \\ Citación: \\ Muñoz-Cedeño R, Martínez P, Paullán-Sani \\ V, Rodríguez-Chica G. Caracterización \\ clínica, histológica y endoscópica del cáncer \\ gástrico en el Hospital de Especialidades Dr. \\ Abel Gilbert Pontón, Ecuador. Rev Colomb \\ Gastroenterol. 2021;36(1):163-171. https://doi. \\ org/10.22516/25007440.558 \\ Médico gastroenterólogo, Hospital de \\ Especialidades Dr. Abel Gilbert Pontón. \\ Guayaquil, Ecuador \\ Médica gastroenteróloga, jefe de servicio de \\ gastroenterología, Hospital de Especialidades \\ Dr. Abel Gilbert Pontón. Guayaquil, Ecuador. \\ Médica gastroenteróloga, médica tratante de \\ gastroenterología y endoscopia digestiva. \\ Guayaquil, Ecuador. \\ ${ }^{4}$ Médico general, residente en el Hospital \\ Universitario de Guayaquil. Guayaquil, Ecuador. \\ *Correspondencia: Rubén Gustavo Muñoz-Cedeño. \\ drubenmunoz@gmail.com \\ Fecha recibido: $\quad 15 / 06 / 20$ \\ Fecha aceptado: 26/01/21
}

\begin{abstract}
Resumen
Introducción: el cáncer gástrico, a nivel mundial, tiene una incidencia variable y es una de las causas más frecuentes de muerte. En Ecuador ocupa el segundo lugar de muerte en los hombres y la cuarta en las mujeres por cáncer gástrico. Objetivos: establecer las características sociodemográficas, clínicas, histológicas y endoscópicas, y determinar una correlación entre la localización e histología en una población de pacientes con cáncer gástrico en el Hospital de Especialidades de Guayaquil, Dr. Abel Gilbert Pontón. Materiales y métodos: estudio de prevalencia analítico y prospectivo. Se incluyeron las endoscopias digestivas altas de consulta externa y emergencia con signos de sospecha de cáncer gástrico realizadas en el Hospital de Especialidades de Guayaquil, Dr. Abel Gilbert Pontón, Ecuador, entre enero de 2018 y diciembre de 2019. Resultados: en el estudio se incluyeron 62 pacientes con diagnóstico de adenocarcinoma gástrico, el sexo masculino representó el 72,6 \% en relación con el sexo femenino, con el 27,4\%; el rango de edad fue entre los 27 y 95 años, el promedio de edad es de 60,96 \pm 15,1 y la edad de mayor presentación fue de 60 a 70 años. El síntoma que predominó fue el dolor, en un $98,4 \%$, y la pérdida de peso, en un $64,5 \%$; su localización más frecuente fue el antro (50,0\%), su morfología de mayor presentación es el Borrmann tipo III y, con respecto al tipo histológico, se encontró el tipo intestinal $(64,5 \%)$ y el difuso $(29,0 \%)$. El tipo intestinal se presentó en edad más avanzada en un $60 \%-69 \%$, fue más frecuente en el cuerpo $(71,4 \%$ y su localización fue más proximal, en comparación con el tipo difuso, que se presentó más en edad temprana (27-39 años), fue más frecuente en el antro (32,3\%) y su localización fue más distal. Conclusiones: el cáncer gástrico se diagnostica en estadios avanzados, más en hombres que en mujeres y se puede afirmar que en nuestro hospital la localización del cáncer gástrico tipo intestinal se presentó en edades avanzadas, más frecuentemente a nivel proximal y en la clasificación del cáncer gástrico avanzado Borrmann tipo III, lo cual podría tener influencia en el tratamiento y pronóstico. Además, los resultados obtenidos justifican la implementación de programas de detección oportuna y tratamiento de esta grave enfermedad.
\end{abstract}

Palabras clave

Cáncer gástrico, tumor gástrico, neoplasias gástricas, adenocarcinoma gástrico, histología gástrica.

\section{INTRODUCCIÓN}

La International Agency for Research on Cancer (GLOBOCAN, que es una plataforma web interactiva desarrollada por el Centro Internacional de Investigadores sobre el Cáncer [IARC]) reportó la cantidad estimada de casos nuevos en 2018 a nivel mundial, en los que el cáncer de estómago ocupa el quinto lugar, con 1033701 casos $(5,7 \%)(1,2)$. Los países de Asia Oriental, Europa oriental y América del Sur tienen las tasas más altas de incidencia de cáncer gástrico, mientras que América del Norte y partes de África tienen las tasas más bajas, lo que demuestra que varían entre las regiones geográficas, y más del $70 \%$ ocurre en países en vías de desarrollo (3). 


\begin{abstract}
Introduction: Gastric cancer is one of the most common causes of death worldwide, with a varying incidence. In Ecuador, it is the second leading cause of death in men and the fourth in women. Objectives: To establish the socio-demographic, clinical, histological, and endoscopic characteristics of patients with gastric cancer and to determine the correlation between location and histology in this population treated at the Hospital de Especialidades Guayaquil Dr. Abel Gilbert Pontón. Materials and methods: Analytical and prospective prevalence study. Outpatient and emergency upper gastrointestinal endoscopies with signs of suspected gastric cancer performed at the Hospital de Especialidades Guayaquil Dr. Abel Gilbert Pontón - Ecuador between January 2018 through December 2019 were included. Results: The study included 62 patients diagnosed with gastric adenocarcinoma. $72.6 \%$ were male and $27.4 \%$ were female; the age range was between 27 and 95 years, with an average of $60.96 \pm 15.1$ years, the age of onset being between 60 and 70 years. Pain was the most frequent symptom in $98.4 \%$ of cases, followed by weight loss in $64.5 \%$. The antrum was the most common site of cancer (50.0\%), and Borrmann type III was the most common morphology. Intestinal cancer was found in $64.5 \%$ of cases, while diffuse gastric cancer was found in $29.0 \%$. Intestinal cancer was more common in older ages (60-69\%) and the most frequent site of presentation was the body of the stomach $(71.4 \%)$ with a proximal location. In contrast, diffuse gastric cancer was more frequent in younger patients aged between $27-39$ years, more often in the antrum $(32.3 \%)$ at a more distal location. Conclusions: Gastric cancer is more often found in men and is usually diagnosed in advanced stages. Intestinal gastric cancer was most commonly seen at advanced ages in our hospital, most frequently at the proximal site and in the Borrmann type III according to the classification of advanced gastric cancer, affecting its treatment and prognosis. The results obtained support the implementation of programs to diagnose and treat this severe disease in a timely manner.
\end{abstract}

\title{
Keywords
}

Gastric cancer; Gastric tumor; Gastric neoplasms; Gastric adenocarcinoma; Gastric histology.

En América Latina y el Caribe, el cáncer gástrico corresponde al $11 \%$ de los casos nuevos de cáncer y el $18 \%$ de la mortalidad por cáncer. En Norteamérica, el cáncer de estómago corresponde al $3 \%$ de los casos de cáncer y al $4 \%$ de mortalidad por cáncer. En América del Sur, los países con mayor incidencia del cáncer gástrico son Chile, Costa Rica y Colombia. Las tasas estimadas de mortalidad estandarizadas por edad para hombres por cada 100000 son elevadas en Honduras (22,3), Costa Rica (16,8), Perú $(18,2)$, Chile $(15,0)$ y Ecuador $(20,7)$, en comparación con tasas más bajas observadas en algunas regiones de América Latina, como Argentina $(5,6)$ y México $(6,7)(4)$. América Latina tiene una alta prevalencia de infección por Helicobacter pylori y algunas de las tasas de incidencia de cáncer gástrico más altas del mundo (5).

Ecuador en el GLOBOCAN del 2018 se ubicó en el tercer lugar, con 2589 casos (9,2\%) en todo el país; en cuanto al sexo, en el masculino ocupa el segundo lugar, con 1364 casos (10,7\%), y en las mujeres ocupa el cuarto lugar, con 1225 casos (8\%). La mortalidad del cáncer gástrico a nivel mundial ocupa el tercer lugar, con 782685 casos $(8,2 \%)$ $(1,2)$. El Instituto de Lucha contra el cáncer del Ecuador (SOLCA) y el Instituto Ecuatoriano de Estadísticas y Censos (INEC) reportaron, respecto al fallecimiento, que el cáncer gástrico representa la primera causa de muerte de todos los tipos de cánceres (11,58 \%). De los 3291 casos de cáncer reportados en ese año, 234 fallecieron; es decir, el 7,11\%. La provincia con el mayor número de casos fue Pichincha, con 944 (28,68\%); seguida de Guayas, con 428 casos (13,01\%); Manabí, con 329 (10\%); Azuay, con 322 casos $(9,78 \%)$ y Loja, con 275 casos $(8,36 \%)(6-8)$.

Corral y colaboradores publicaron en Quito un estudio en 2018 sobre el cáncer gástrico, en el que se describió una disminución de la incidencia en 1985-1988, que era de $28,5 \%$ en hombres y del $21,7 \%$ en mujeres, comparada con un descenso en los años 2009-2013, que fue del 20,3\% en hombres y en mujeres de $14,5 \%$, con un intervalo de confianza (IC) del $95 \%$ : $-2,7$ a -0,5; y la mortalidad decreciente en 1985-1988 fue del 16,7 \% en hombres y del 13,8\% en mujeres, comparada con un descenso en 2009-2013 de $15,2 \%$ en hombres y del 10,0 \% en mujeres, con un IC $95 \%$ : $-1,7$ a -0,1. La disminución, tanto en incidencia como en mortalidad, probablemente está relacionada con las mejores condiciones socioeconómicas, mejoramiento de la calidad de la alimentación y de su preservación, antes que la intervención de los servicios de salud en el diagnóstico temprano, y se concluye que desde 1998 hasta 2013 la disminución fue significativa (CPA: -2,4; IC $95 \%: 3,8-1,1$ ) tanto en hombres como en mujeres (9).

El cáncer gástrico es una enfermedad heterogénea que afecta a personas con edad avanzada entre la sexta y séptima décadas de la vida (el $80 \%$ de los casos se diagnostica en 
mayores de 65 años), con predominio en varones y estratos socioeconómicos de bajo nivel (10). Los factores de riesgo del cáncer gástrico son consistentes con la patogénesis multifactorial como el tabaquismo, el consumo de alcohol, el alto consumo de carnes rojas o carne procesada y el consumo excesivo de sal, que se asociaron con aumento moderado en el riesgo de cáncer gástrico. En cambio, los niveles altos de educación, el consumo de frutas y consumo total de vegetales se asociaron con un riesgo moderadamente reducido para cáncer gástrico (11). La prevalencia de infección por $H$. pylori y otros factores de riesgo pueden variar la tasa de incidencia del cáncer gástrico en un estudio publicado por Sierra y colaboradores en 2016, en el que se indica que los países como Chile, Costa Rica, Colombia, Ecuador, Brasil y Perú los hombres tenían 3 veces más altas las tasas de incidencia y mortalidad estandarizadas por edad que las mujeres (12).

Mundialmente, se reconoce a la infección de $H$. pylori como la causa primaria del cáncer gástrico, pero se han identificado también varios factores de riesgo como el nivel socioeconómico, la dieta alta en sal y baja en antioxidantes, y el consumo de alcohol y tabaco. En Ecuador confluyen múltiples variables geográficas, físicas y biológicas, pero también sociales, económicas y culturales que podrían estar relacionadas con la ocurrencia y el curso de la enfermedad; esto se debe a que la asociación de factores de riesgo para el cáncer gástrico en América Latina se basa en comparaciones de casos y controles que tienen una confiabilidad incierta, particularmente con respecto a la dieta $(11,13)$.

La histología según Lauren se divide en los tipos intestinal y difuso, que pueden presentar diferencias epidemiológicas, fisiopatológicas y pronósticas, las cuales pueden condicionar el abordaje diagnóstico y terapéutico, y en un $10 \%$ a $20 \%$ puede existir la forma indiferenciada o inclasificable. Anatómicamente, se distinguen 2 regiones que se pueden clasificar en estómago proximal (fondo y cuerpo proximal) y estomago distal (cuerpo distal, antro y píloro). El desarrollo del cáncer gástrico es un proceso multifactorial, complejo y de larga evolución (10).

Los objetivos del presente estudio son establecer las características sociodemográficas, clínicas, histológicas y endoscópicas, y determinar la localización en relación con la histología en una población de pacientes con cáncer gástrico en el Hospital de Guayaquil.

\section{MATERIALES Y MÉTODOS}

El presente estudio es de prevalencia analítica, correlacional de tipo corte transversal, prospectivo realizado durante el período comprendido entre enero de 2018 y diciembre de 2019 , con los pacientes hospitalizados en la unidad de gastroenterología del Hospital de especialidades de Guayaquil, Dr. Abel Gilbert Pontón, Ecuador, la cual es una institución de tercer nivel adscrita al posgrado de Gastroenterología de la Universidad de Guayaquil.

El objetivo principal del estudio fue establecer las características sociodemográficas, clínicas, histológicas y endoscópicas del cáncer gástrico en los pacientes sometidos a endoscopia digestiva alta con signos de sospecha de cáncer de la consulta externa y de emergencias. La población de estudio corresponde a pacientes adultos a quienes se les realizó endoscopia digestiva alta y se observó una lesión neoplásica según la clasificación de Borrmann, con un resultado histopatológico según la clasificación de Lauren y que, además, aceptaron participar en el estudio.

Los criterios de exclusión fueron pacientes ya diagnosticados de cáncer gástrico, antecedentes de haber sido operados por cáncer gástrico, pacientes sin resultado histopatológico o con un resultado extraviado, $u$ otro tipo de neoplasias diferentes al adenocarcinoma (como neuroendocrino, linfoma, tumores del estroma gastrointestinal, entre otros), cáncer localizado en la unión gastroesofágica y aquellos que no aceptaron participar en el estudio.

Dentro de las variables incluidas en el estudio se encuentran la edad, sexo del paciente, tipo histológico según la clasificación de Lauren (difuso e intestinal) y grado histológico (diferenciado, moderadamente diferenciado e indiferenciado). Con base en las características endoscópicas, se determinó la localización en relación con el punto de extensión más alto (fondo, cuerpo y antro) y la morfología en relación con la clasificación de Borrmann (I, II, III y IV).

La recolección de los datos se realizó mediante el instrumento elaborado con base en las variables del estudio. La digitalización de los datos se realizó con el programa Excel 8.0 y el análisis, con IBM SPSS Statistics 22. Las variables continuas se expresaron con la media \pm desviación estándar $(\mathrm{DE})$ y las variables discretas se expresaron en frecuencia y proporciones. También se realizó un análisis bivariado entre algunas variables de interés, utilizando la prueba de chi cuadrado $\left(\chi^{2}\right)$.

El estudio no involucró ningún riesgo para los pacientes, se hizo empleando la información rutinaria del servicio de gastroenterología previo consentimiento informado y manteniendo los datos de forma confidencial.

\section{RESULTADOS}

Durante el período de estudio de enero de 2018 a diciembre del 2019, se encontraron 68 pacientes con tumor gástrico, de los que se excluyeron 6 pacientes que no cumplían los criterios de inclusión: 4 por linfomas gástricos, un tumor estromal gastrointestinal y un tumor neuroendocrino. Finalmente, el estudio se realizó con 62 pacientes con diagnóstico de adenocarcinoma gástrico; el sexo masculino representó el $72,6 \%$ con 45 casos y el sexo femenino, el $27,4 \%$ con 17 casos, correspondiendo una razón 
de hombre: mujer de 2,64: 1,00. Los pacientes estuvieron en un rango de edad entre los 27 y 95 años, el promedio de edad fue de $60,96 \pm 15$,1 años y la mayoría estaba distribuida entre los 60-79 y 40-59 años de edad, con 53,2 \% (33 casos) y 27,4\% (17 casos), respectivamente (Tabla 1 ). En cuanto a los hábitos, el alcohol tuvo una relación con el cáncer gástrico en un 43,5\%, con 27 casos; y el cigarrillo, en $30,6 \%$, con 19 casos; y con respecto a los antecedentes familiares de patologías de cáncer, solo el $5 \%$ presentó cáncer gástrico familiar en primer grado de consanguinidad ( 1 caso) y en segundo grado de consanguinidad ( 2 casos). Adicionalmente, los síntomas más frecuentes fueron el dolor $(98,4 \%)$, pérdida de peso $(64,5 \%)$, vómitos $(40,3 \%)$, melena $(38,7 \%)$ y hematemesis $(16,1 \%)$ (Tabla 1$)$.

Tabla 1. Características sociodemográficas y clínicas

\begin{tabular}{lcc|}
\hline \multicolumn{1}{c}{ Variables } & Frecuencia & Porcentaje \\
\hline Sexo & 17 & \\
- Femenino & 45 & 72,4 \\
- Masculino & & \\
Edad & $60,96 \pm 15,1$ & \\
- Media \pm DE & $27-95$ & \\
- Rango & 6 & 9,7 \\
\hline - $\leq 39$ & 17 & 27,4 \\
\hline - 40-59 & 33 & 53,2 \\
\hline - $60-79$ & 6 & 9,7 \\
\hline - > 80 & & \\
\hline Hábitos & 19 & 30,6 \\
\hline - Cigarrillo & 27 & 43,5 \\
\hline - Alcohol & & \\
\hline Características clínicas & 61 & 98,4 \\
\hline - Dolor & 40 & 64,5 \\
\hline - Pérdida de peso & 25 & 40,3 \\
\hline - Vómitos & 24 & 38,7 \\
\hline - Melena & 10 & 16,1 \\
\hline - Hematemesis & &
\end{tabular}

En cuanto a la localización del cáncer, el $50 \%$ se localizó en el antro; 45,2 \% en el cuerpo y 4,8 \% en el fondo. De acuerdo con la morfología, según la clasificación de Borrmann, se encontró que el 6,5 \% era de tipo Borrmann I; 19,4 \%, de tipo Borrmann II; 62,9 \%, de tipo Borrmann III; y $11,3 \%$, de tipo Borrmann IV. Con respecto al tipo histológico según la clasificación de Lauren, se hallaron pacientes con cáncer gástrico de tipo intestinal: 40 (64,5\%), tipo difuso: 19 (29,0 \%) y tipo mixto: 4 (6,5\%).

Con el propósito de correlacionar la histología según Lauren y la localización del tumor, se encontró que el tipo intestinal se localiza más frecuentemente en el cuerpo (20 casos, $71,4 \%$ ), mientras que el difuso se localiza en el antro (10 casos, 32,3\%); y en la correlación de la histología con el tipo de Borrmann, el tipo III es de mayor frecuencia en los pacientes con cáncer gástrico intestinal ( 24 casos, $61,5 \%$ ) que en el difuso (13 casos, 33,3\%), y se realizó un análisis bivariado con las variables de grado histológico y morfología tipo Borrmann, con una correlación de intervalo ( $\mathrm{r}$ $=0,312$ ), el tipo histológico se correlaciona con el grado histológico en una correlación de intervalo $(\mathrm{r}=0,453)$ y se encontró una correlación de Pearson significativa en los niveles de 0,05 y 0,01 (Tabla 2).

Tabla 2. Características endoscópicas e histológicas de acuerdo con los tipos histológicos del cáncer gástrico en el Hospital de Especialidades Dr. Abel Gilbert Pontón

\begin{tabular}{lcccc} 
& \multicolumn{3}{c}{ Histopatología, n (\%) } & Total \\
\cline { 2 - 4 } & Difuso & Intestinal & Mixto & \\
\hline Localización & & & & \\
- Antro & $10(32,3)$ & $18(58,1)$ & $3(9,7)$ & $31(50,0)$ \\
\hline - Cuerpo & $7(25,0)$ & $20(71,4)$ & $1(3,6)$ & $28(45,2)$ \\
\hline - Fondo & $1(33,3)$ & $2(66,7)$ & $0(0,0)$ & $3(4,8)$ \\
\hline Morfología & & & & \\
\hline - Borrmann I & $1(25,0)$ & $3(75,0)$ & $0(0,0)$ & $4(6,5)$ \\
\hline - Borrmann II & $1(8,3)$ & $9(72,0)$ & $2(16,7)$ & $12(19,4)$ \\
\hline - Borrmann III & $13(33,3)$ & $24(61,5)$ & $2(5,1)$ & $39(62,9)$ \\
\hline - Borrmann IV & $3(42,9)$ & $4(57,1)$ & $0(0,0)$ & $7(11,3)$ \\
\hline Grado histológico & & & & \\
\hline - Diferenciado & $0(0,0)$ & $6(75,0)$ & $2(25,0)$ & $8(12,9)$ \\
\hline - Moderadamente & $6(20,0)$ & $22(73,3)$ & $2(6,7)$ & $30(48,4)$ \\
\hline diferenciado & $12(50,0)$ & $12(50,0)$ & $0(0,0)$ & $24(38,7)$ \\
\hline - Indiferenciado & & & & \\
\hline
\end{tabular}

En cuanto al grado de diferenciación, fue diferenciado en 8 casos $(12,9 \%)$, moderadamente diferenciado en 30 $(48,4 \%)$ e indiferenciado en 24 (38,7\%); además, se halló que el $75 \%$ de los diferenciados y el 73,3 \% de los moderadamente diferenciados eran pacientes con cáncer gástrico de tipo intestinal, y en los indiferenciados no hubo diferen- 
cia con un $50 \%$ para los tipos intestinal y difuso, como se observa en la Tabla 2.

En las Figuras 1 y 2 se representa la distribución del tipo de histología según Lauren por grupo etario y sexo. En el sexo masculino, el tipo intestinal es de $31(77,5 \%)$ y en el sexo femenino, el tipo difuso es de $8(44,4 \%)$ y el intestinal es de $9(22,5 \%)$. La razón hombre: mujer para el tipo intestinal fue 3,44: 1,00 (31/9) y para el difuso fue 1,25: 1,00 , $(10 / 8)$. En los hombres, se observó un incremento del adenocarcinoma de tipo intestinal desde los 50 años con un pico máximo en edades de 60-69 años; mientras que el tipo difuso se mantiene (Figura 1). En las mujeres se observó que en el tipo difuso se presentó un aumento en edades tempranas (de 27 a 39 años de edad), y se mantiene conforme aumenta la edad; a diferencia del tipo intestinal, en el que se observa un aumento entre los 60 y 69 años de edad, período en el que se presentó un pico máximo (Figura 2).

\section{DISCUSIÓN}

El cáncer gástrico en Ecuador, en un análisis epidemiológico en el Hospital Carlos Andrade Marín en Quito, Ecuador, demostró que la prevalencia del cáncer gástrico es de $61 \%$ en hombres y de $39 \%$ en mujeres, con un promedio de edad de 68 años para ambos sexos, y los factores asociados son $H$. pylori (46\%), alcohol (36\%) y tabaco
(35\%) (14). En México se encuentra entre las principales causas de morbilidad hospitalaria en los varones; la tasa más alta se ubica en la población de 75-79 años (47 de cada 100000 hombres de ese grupo de edad), seguidos por la población de 64-74 años (38 de 100000 del mismo grupo de edad). Actualmente se sabe que uno de los principales tumores malignos por los que fallece la población mexicana es el cáncer gástrico, que es la tercera causa tanto en la población femenina ( $7 \%$ ) como en la masculina $(8,6 \%)(10)$. Novo y colaboradores en su estudio indicaron que el $75 \%$ de los casos de cáncer gástrico se presenta en los hombres y el $25 \%$ en las mujeres, principalmente con edades entre 70 y 80 años (50\%), y entre 60 y 70 años (34\%) (15). Lo anterior coincide con nuestro estudio, en el que el sexo masculino es el más afectado, con el 72,6\% de los casos, a diferencia del sexo femenino, con $27,4 \%$ de los casos, con una razón de hombre: mujer de 2,64: 1,00, como los estudios ya mencionados.

Los síntomas y signos habituales son la saciedad precoz, pérdida de peso y de apetito, dolor epigástrico y vómitos; los adenocarcinomas avanzados ulcerados pueden manifestarse con hematemesis o melena, y si afecta el antro-píloro, puede ocasionar un obstáculo en la salida del contenido gástrico al duodeno y provocar un síndrome pilórico (16). Nuestro estudio mostró que el síntoma más frecuente fue el dolor $(98,4 \%)$ seguido de la pérdida de peso $(64,5 \%)$,

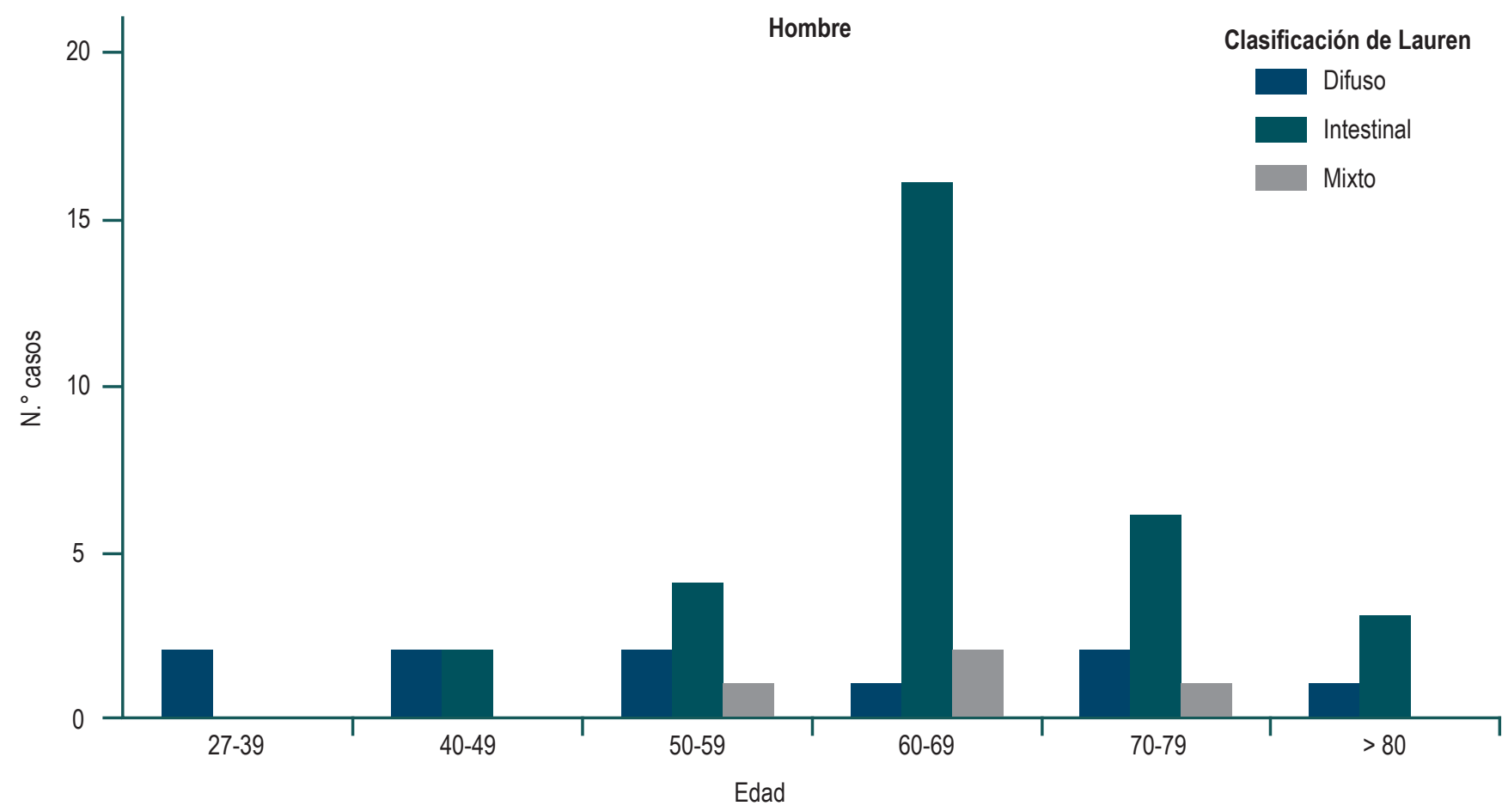

Figura 1. Distribución de tipos histológicos por grupos etarios en hombres. 


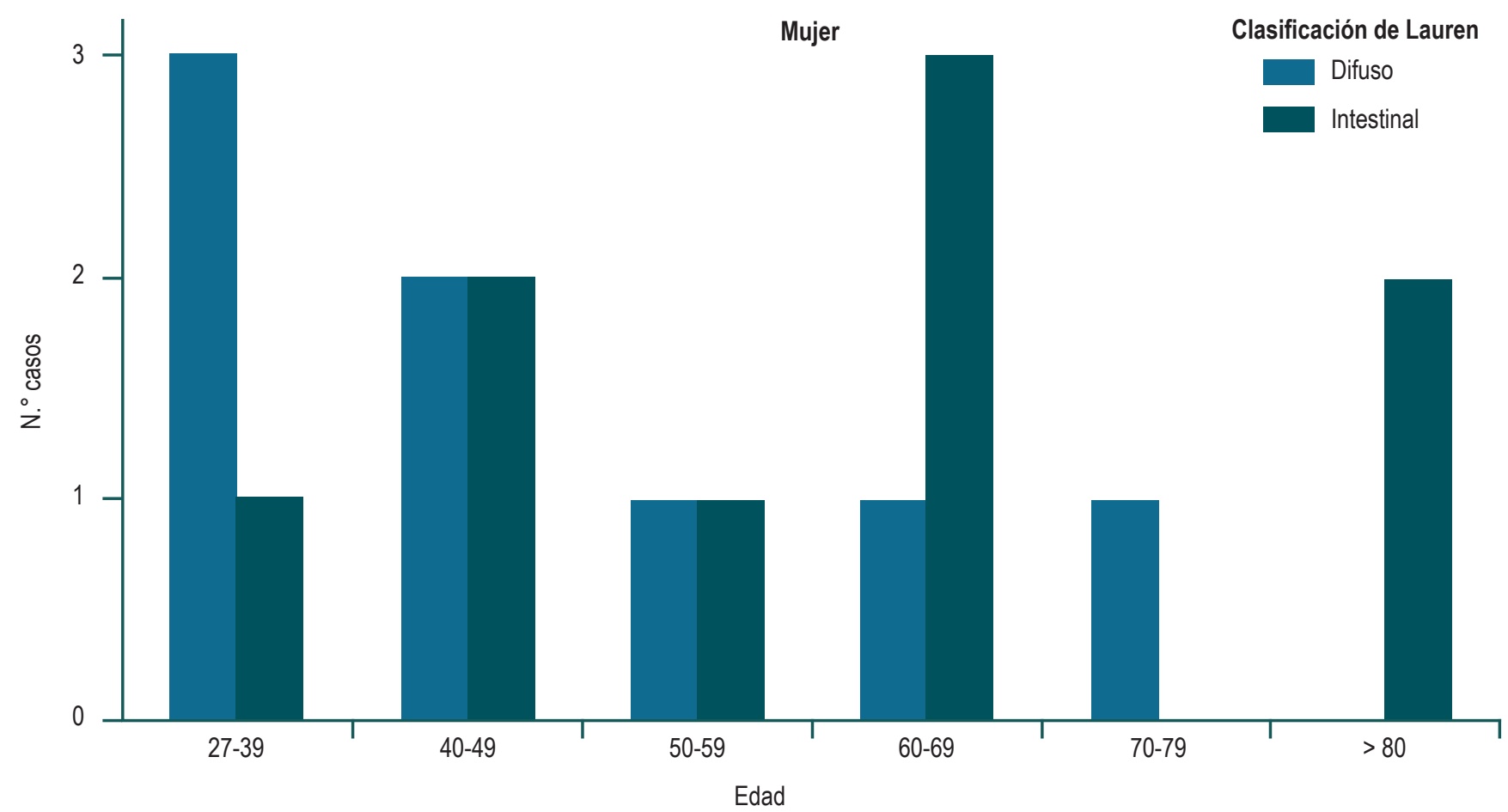

Figura 2. Distribución de tipos histológicos por grupos etarios en mujeres.

vómitos y melena (menos del $40 \%$ ), lo que coincide con estudios como el de Sánchez y colaboradores, quienes indicaron que los síntomas del cáncer gástrico no son específicos y pueden imitar otras enfermedades gastroduodenales; los síntomas más habituales fueron el dolor (70\%), anorexia, náuseas y pérdida de peso (50\%), disfagia $(20 \%)$ y hemorragia gastrointestinal (5\%) (17).

La localización del cáncer gástrico ocurre entre un $40 \%$ y $60 \%$ en el antro-píloro, un $20 \%-25 \%$ en el cuerpo y un $15 \%-20 \%$ en el fondo; y el tipo histológico intestinal es el más frecuente (16), lo que coincide con nuestro estudio, en el que el cuerpo y antro presentaron igual porcentaje, pero hubo una diferencia en el porcentaje del fondo en menor proporción (de 4,8\%), diferencia que se podría deber al tamaño muestral. Con respecto a la histología, se han observado diferencias clínicas entre los tipos de cáncer, ya sea de tipo difuso o intestinal (según la clasificación de Lauren). El tipo difuso ocurre en todos los grupos de edad con distribución similar con respecto al sexo, a diferencia del intestinal, que predomina en los hombres y en las personas de edad avanzada. El tipo difuso se encuentra en el cuerpo del estómago, en tanto que el intestinal predomina más en el antro y en la incisura. Adicionalmente, el tipo difuso tiene tendencia a invadir la pared gástrica y producir metástasis, además de que tiene una progresión más rápida y un pronóstico más pobre (18). Con el propósito de correlacionar la histología según la clasificación de Lauren y la localización del tumor, en nuestro estudio se encontró que el tipo intestinal predomina más en hombres, con 31 casos (77,5 \%), y se localizó en el cuerpo en 20 casos (71,4\%); y el difuso predominó en mujeres, con 8 casos $(44,4 \%)$ y se localizó más en el antro, con 10 casos $(32,3 \%)$; en este aspecto se pudieron encontrar diferencias con estudios ya mencionados, que podrían estar relacionados con el tamaño muestral y la duración de nuestro estudio.

En Ecuador, Andrade y colaboradores demostraron que los tipos de cáncer gástrico más frecuentes, según la clasificación de Lauren, fueron el adenocarcinoma difuso (60\%) y el adenocarcinoma de tipo intestinal (40\%) (14). Borch y colaboradores mencionaron que el tipo intestinal representó el 52,7 \% de los casos, seguido del difuso, con $33,4 \%$, y del mixto, con un 13,9\% (19). Martínez-Galindo y colaboradores publicaron en México en 2015 un estudio con 415 casos de adenocarcinoma gástrico en el que 230 casos $(55,2 \%)$ fueron de tipo difuso y la edad media fue de 54,02 años, de los cuales el 51,3\% eran hombres, 118 casos (el 28,2 \%) eran de tipo intestinal y la edad media de presentación fue de 63,43 años (10). Existen grandes diferencias tanto en regiones como en países con respecto al tipo histológico más frecuente, que nuestro estudio fue el tipo intes- 
tinal (64,5\%), seguido del difuso (29\%), lo que se ha demostrado en estudios ya publicados, como el de Borch y colaboradores (19), y Marín y colaboradores (20); pero en estudios recientes como el de Martínez-Galindo y colaboradores (10), y Quiñones y colaboradores (21), el tipo difuso fue el más frecuente. En Ecuador, Andrade y colaboradores demostraron que el tipo difuso es el más frecuente, lo que se debe a la disminución de la incidencia de cánceres de tipo intestinal o, en nuestro país, al cambio de provincias en Ecuador que pueden ser estudiadas en otro trabajo, debido a los pocos estudios que existen en nuestro país.

El estudio por Quiñones y colaboradores, publicado en 2011, demostró que el tipo difuso era másfrecuente (51,5\%), seguido del intestinal (33,9\%) en Perú, y se encontró una asociación entre el tipo intestinal con la localización distal $(58,3 \%$ frente a $44,1 \%, p=0,004)$, y el tipo difuso con localización proximal $(19,3 \%$ frente a $12,4 \% ; p=0,049)(21)$. Marín y colaboradores publicaron los tipos de neoplasias proximales; los tipos intestinal (51\%) y difuso (49\%) son casi iguales; mientras que para las localizaciones distales, el predominio es más de tipo intestinal, con $64 \%$, a diferencia del difuso, con $36 \%$ (20); estos hallazgos coinciden con nuestro estudio, en el que el tipo intestinal fue más proximal $(71,4 \%)$ y se presentó más en hombres; mientras que el difuso fue más distal $(32,4 \%)$ en mujeres, a diferencia del estudio de Quiñones y colaboradores (21).

Andrade y colaboradores demostraron que, según la etapa, el $84 \%$ se atendió en etapa avanzada, en la que se encontró que el Borrmann tipo III o ulcerado infiltrativo fue el más frecuente, con $42 \%$; seubicaba principalmente en el antro (39\%), seguido del cuerpo $(22,7)$, con localización distal del estómago en $61 \%$ en el Hospital de Especialidades Carlos Andrade Marín en Quito, Ecuador (14). Adrada y colaboradores publicaron un estudio en Colombia, en el departamento de Cauca entre los años 2003 y 2006, con 225 casos de cáncer gástrico, de los cuales el $66 \%$ era en hombres, $84 \%$ ancianos, con localización distal en el 68 \% de los casos, de características avanzadas en $92 \%$ y con clasificación macroscópica de Borrmann III el $58 \%$ y Borrmann IV el 23\%; la histología mostró que el $79 \%$ era de tipo intestinal y el $21 \%$ de tipo difuso (22). Los resultados del anterior estudio coinciden con el nuestro, en que el tipo Borrmann III es el más frecuente $(62,9 \%)$ y, según el tipo histológico, el intestinal fue el de mayor frecuencia $(61,5 \%)$.

En un análisis epidemiológico de cáncer gástrico en Ecuador, según el grado histológico, el diferenciado fue del $15 \%$, el moderadamente diferenciado fue del $25 \%$, el pobremente diferenciado fue del $45 \%$ y el indiferenciado fue del $15 \%$ (14). Nakamura y colaboradores (23) mencionaron que el cáncer gástrico diferenciado es más de tipo intestinal y que el indiferenciado o pobremente indiferenciado es más de tipo difuso (24).

No obstante, deberían realizarse estudios prospectivos, de mayor duración, con mayor tamaño muestral y multicéntricos con fin de poder garantizar los hallazgos encontrados en nuestro estudio. Además, nuestro hospital es un centro de referencia a nivel de la provincia del Guayas, por lo que los presentes hallazgos no podrían ser generalizados a toda la población ecuatoriana, pero sí podrían ser tomados en cuenta para dar un acercamiento de las características de los pacientes con cáncer gástrico en la provincia, debido a que este es un hospital de referencia del Ministerio de Salud en dicha región. Como limitaciones hubo falta de patólogos especializados en nuestra unidad para reportar los resultados en los casos cuyos reportes histopatológicos tuvieron un tiempo de demora de más de 15 a 30 días.

\section{CONCLUSIONES}

El cáncer gástrico se diagnostica en estadios avanzados, es más frecuente en hombres que en mujeres y la edad de mayor presentación es de 60 a 70 años. Hubo una relación con el consumo de alcohol y tabaco como factor de riesgo, y los síntomas de mayor predomino fueron el dolor y la pérdida de peso. Su localización más frecuente fue en el antro y el tipo más frecuente de cáncer gástrico fue el de tipo intestinal, seguido del difuso; el intestinal se presentó en una edad más avanzada, es más frecuente en el cuerpo y su localización fue proximal; mientras que el tipo difuso se presentó más en edad temprana, es más frecuente en el antro y su localización fue distal. Se puede afirmar que en nuestro hospital la localización del cáncer gástrico de tipo intestinal se presentó en edad avanzada, más frecuentemente a nivel proximal y en la clasificación del cáncer gástrico avanzado según Borrmann tipo III, lo cual podría tener influencia en el tratamiento y pronóstico. Además, los resultados obtenidos justifican la implementación de programas de detección oportuna y tratamiento de esta grave enfermedad.

\section{Confidencialidad de los datos}

Los autores declaran que han seguido los protocolos de su centro de trabajo sobre la publicación de datos de pacientes.

\section{Derecho a la privacidad y consentimiento informado}

Los autores han obtenido el consentimiento informado de los pacientes o sujetos referidos en el artículo. Este documento obra en poder del autor de correspondencia. 
Fuentes de financiación

Propias de los autores.

\section{Conflictos de interés}

Los autores declaran no tener ningún conflicto de interés.

\section{REFERENCIAS}

1. Cancer today [internet]. WHO, IARC; 2020 [consultado el 26 de abril de 2020]. Disponible en: https://gco.iarc.fr/ today $/$ online-analysis-pie $\mathrm{v}=2020 \&$ mode $=$ cancer\&mode population $=$ continents \&population $=900 \&$ populatio $\mathrm{ns}=900 \& \mathrm{key}=$ total $\&$ sex $=0 \&$ cancer $=39 \&$ type $=0 \&$ sta tistic $=5 \&$ prevalence $=0 \&$ population_group $=0 \&$ ages group $\% 5 \mathrm{~B} \% 5 \mathrm{D}=0$ \&ages_group $\% 5 \mathrm{~B} \% 5 \mathrm{D}=17 \& \mathrm{nb}$ items $=7$ \&group_cancer $=1$ \&include_nmsc $=1$ \&include nmsc_other $=1$ \& half_pie $=0$ \&donut $=0$

2. Ecuador [internet]. WHO, IARC; 2020 [consultado el 26 de abril de 2020]. Disponible en: https://gco.iarc.fr/ today/data/factsheets/populations/218-ecuador-factsheets.pdf

3. Jemal A, Bray F, Center MM, Ferlay J, Ward E, Forman D. Global cancer statistics. CA Cancer J Clin. 2011;61(2):69-90. https://doi.org/10.3322/caac.20107

4. Torres J, Correa P, Ferreccio C, Hernandez-Suarez G, Herrero R, Cavazza-Porro M, et al. Gastric cancer incidence and mortality is associated with altitude in the mountainous regions of Pacific Latin America. Cancer Causes Control. 2013 Feb;24(2):249-56. https://doi.org/10.1007/s10552-012-0114-8

5. Coelho LG, León-Barúa R, Quigley EM. Latin-American Consensus Conference on Helicobacter pylori infection. Latin-American National Gastroenterological Societies affiliated with the Inter-American Association of Gastroenterology (AIGE). Am J Gastroenterol. 2000;95(10):2688-91. https://doi.org/10.1111/j.1572-0241.2000.03174.x

6. Camas y Egresos Hospitalarios [internet]. INEC; 2019 [consultado el 26 de abril de 2020]. Disponible en: https://www.ecuadorencifras.gob.ec/camas-y-egresoshospitalarios/

7. Registro de Tumores SOLCA matriz [internet]. SOLCA; 2013 [consultado el 26 de abril de 2020]. Disponible en: http://www.estadisticas.med.ec/webpages/graficos.jsp

8. Estrategia nacional para la atención integral del cáncer en el Ecuador [internet]. Ministerio de Salud Pública del Ecuador; 2017 [consultado el 26 de abril de 2020]. Disponible en: https://aplicaciones.msp.gob.ec/salud/ archivosdigitales/documentosDirecciones/dnn/archivos/ ac_0059_2017.pdf

9. Corral Cordero F, Cueva Ayala P, Yépez Maldonado J, Tarupi Montenegro W. Trends in cancer incidence and mortality over three decades in Quito - Ecuador. Colomb
Med (Cali). 2018;49(1):35-41. https://doi.org/10.25100/cm.v49i1.3785

10. Martínez-Galindo MG, Zamarripa-Dorsey F, CarmonaCastañeda A, Angeles-Labra A, Peñavera-Hernández R, Ugarte-Briones C, et al. Histopathologic characteristics of gastric adenocarcinoma in Mexican patients: a 10-year experience at the Hospital Juárez of Mexico. Rev Gastroenterol Mex. 2015;80(1):21-6. https://doi.org/10.1016/j.rgmx.2014.11.002

11. Bonequi P, Meneses-González F, Correa P, Rabkin CS, Camargo MC. Risk factors for gastric cancer in Latin America: a meta-analysis. Cancer Causes Control. 2013;24(2):217-31. https://doi.org/10.1007/s10552-012-0110-z

12. Sierra MS, Cueva P, Bravo LE, Forman D. Stomach cancer burden in Central and South America. Cancer Epidemiol. 2016;44 Suppl 1:S62-S73. https://doi.org/10.1016/j.canep.2016.03.008

13. Correa P. Cáncer gástrico: una enfermedad infecciosa. Revista Colombiana de Cirugía. 2011;26(2):111-7.

14. Andrade Díaz C. Análisis epidemiológico del cáncer gástrico en el Hospital de Especialidades Carlos Andrade Marín en el período enero-diciembre del año 2016. Revista Médica-Científica CAMbios HECAM. 2019;17(1):10-4. https://doi.org/10.36015/cambios.v17.n1.2018.4

15. Novo CY, Diermissen RH, Rojas BR. Estudio observacional en pacientes con cáncer gástrico en etapa pre y post quirúrgica desde la perspectiva fisioterapéutica, Hospital México, durante el mes Mayo 2015. Rev Med Cos Cen. 2016;73(621):765-771.

16. Quintero Carrión E, Nicolás Pérez D. Adenocarcinoma gástrico y de la unión esófago-gástrica. Medicine. 2004;9(2):100-11. https://doi.org/10.1016/S0211-3449(04)70235-0

17. Sánchez Gaitán E. Adenocarcinoma gástrico. Revista Médica Sinergia. 2017;2(1):13-21.

18. Lauren P. The two histological main types of gastric carcinoma: diffuse and so-called intestinal-type carcinoma. An attempt at a histo-clinical classification. Acta Pathol Microbiol Scand. 1965;64:31-49. https://doi.org/10.1111/apm.1965.64.1.31

19. Borch K, Jönsson B, Tarpila E, Franzén T, Berglund J, Kullman E, et al. Changing pattern of histological type, location, stage and outcome of surgical treatment of gastric 
carcinoma. Br J Surg. 2000;87(5):618-26.

https://doi.org/10.1046/j.1365-2168.2000.01425.x

20. Marín Martínez JD, Olarte Garzón MA, Rodríguez Lizarazo JI, Gómez Marulanda JC, Villa Molano JC, Tovar Rey MH, et al. Características de los pacientes con cáncer gástrico del departamento de Cundinamarca, remitidos al Hospital Universitario de la Samaritana entre los años 2004 y 2009. Rev Col Gastroenterol. 2010;25(4):334-48.

21. Quiñones J, Portanova M, Yabar A. Relación entre tipo histológico y la localización del adenocarcinoma gástrico en el Hospital Rebagliati. Rev Gastroenterol Perú. 2011;31(2):139-45.

22. Adrada JC, Calambás FH, Díaz JE, Delgado DO, Sierra $\mathrm{CH}$. Características sociodemográficas y clínicas en una población con cáncer gástrico en el Cauca, Colombia. Rev Col Gastroenterol. 2008;23(4):309-14.

23. Nakamura K, Ueyama T, Yao T, Xuan Z, Ambe K, Adachi $\mathrm{Y}$, et al. Pathology and prognosis of gastric carcinoma. Findings in 10,000 patients who underwent primary gastrectomy. Cancer. 1992; 1(70): p. 1030-7.

https://doi.org/10.1002/1097-

0142(19920901)70:5<1030::aidcncr2820700504>3.0.co;2-c

24. Espejo Romero H, Navarrete Siancas J. Clasificación de los adenocarcinomas de estómago. Rev Gastroenterol Perú. 2003;23(1):199-212. 